\title{
Evaluation of Prostate-Specific Membrane Antigen as an Imaging Reporter
}

\author{
Mark A. Castanares ${ }^{1}$, Amarnath Mukherjee ${ }^{2}$, Wasim H. Chowdhury ${ }^{2}$, Minzhi Liu ${ }^{2}$, Ying Chen $^{3}$, Ronnie C. Mease ${ }^{3}$, \\ Yuchuan Wang ${ }^{3}$, Ronald Rodriguez ${ }^{2}$, Shawn E. Lupold*2, and Martin G. Pomper*1,3 \\ ${ }^{I}$ Department of Pharmacology and Molecular Sciences, Johns Hopkins University School of Medicine, Baltimore, Maryland; ${ }^{2}$ James \\ Buchanan Brady Urological Institute and Department of Urology, Johns Hopkins University School of Medicine, Baltimore, \\ Maryland; and ${ }^{3}$ Russell H. Morgan Department of Radiology and Radiological Science, Johns Hopkins University School of \\ Medicine, Baltimore, Maryland
}

\begin{abstract}
Genetic reporters provide a noninvasive method to monitor and evaluate a population of cells. The ideal properties of a gene reporter-probe system include biocompatibility, lack of immunogenicity, low background expression or signal, and high sensitivity of detection. The prostate-specific membrane antigen (PSMA) is an attractive candidate for a genetic reporter as it is a human transmembrane protein with a selective expression pattern, and there are several PSMA imaging agents available for clinical and preclinical applications. We evaluated the use of PSMA as a genetic imaging reporter by comparison to 2 clinically established reporters, the mutant herpes simplex virus type I thymidine kinase and the human sodium-iodide symporter. Methods: Adenoviruses expressing each reporter were constructed and validated in vitro for expression and function. To compare PSMA with existing imaging reporters, a bilateral Matrigel suspension model was established with nude mice bearing cells equally infected with each reporter or control adenovirus. Dynamic PET was performed, and time-activity curves were generated for each reporter-probe pair. Results: A comparison of peak target-to-background ratios revealed that PSMA offered the highest ratio relative to the control Matrigel suspension as well as muscle. Further, as proof of concept, PSMA was applied as an imaging reporter to monitor adenoviral liver transduction with both nuclear and optical imaging probes. Conclusion: These preliminary studies support further development of PSMA as a noninvasive genetic reporter.
\end{abstract}

Key Words: PSMA; molecular-genetic imaging; DCFPyL; positron emission tomography; optical imaging

J Nucl Med 2014; 55:805-811

DOI: 10.2967/jnumed.113.134031

G ene reporter-probe systems offer a noninvasive means to monitor gene therapy and to track the movement of cells or the activation of signal transduction pathways (1). Limitations of current reporter-probe systems for clinical translation include the lack of biocompatibility due to immunogenicity, low sensitivity due to

Received Oct. 21, 2013; revision accepted Jan. 8, 2014.

For correspondence or reprints contact: Martin G. Pomper, Johns Hopkins Medical School, 1550 Orleans St., CRB II, Baltimore, MD 21287.

E-mail: mpomper@jhmi.edu

${ }^{*}$ Contributed equally to this work.

Published online Apr. 3, 2014.

COPYRIGHT (c) 2014 by the Society of Nuclear Medicine and Molecular Imaging, Inc. insufficient reporter expression per cell or lack of a signal amplification mechanism, and high background signal due to nonspecific binding of probe. Despite those hurdles, several reporter genes, including the mutant herpes simplex virus thymidine kinase (HSV-sr39tk) and the human sodium iodide symporter (hNIS), have been applied clinically $(2,3)$. In light of this achievement, there is substantial opportunity to develop new reporter-probe systems based on other human genes (4-6). Several recent reviews have covered this topic $(1,7)$.

The prostate-specific membrane antigen (PSMA) is a human transmembrane protein that possesses many properties that are desirable as a reporter for an imaging system. PSMA is internalized on the binding of certain ligands (8), providing a mechanism to accumulate PSMA-targeting agents within cells. The expression of PSMA is limited to the prostate, the proximal tubules of the kidney, and the brain (9). Alternative processing results in both cell surface and cytoplasmic isoforms, the latter of which is expressed primarily in the healthy prostate (10). PSMA expression is low and isolated in healthy tissues, allowing imaging of cells that express the surface form of PSMA at high levels, such as metastatic prostate tumors (11-13). There are several PSMA-binding ligands that have been developed for the delivery of imaging and therapeutic agents for prostate cancer, including low-molecular-weight nuclear, fluorescent, and multimodality imaging probes $(14,15)$. This combination of tissue-restricted expression, human biocompatibility, available probe diversity, and proven clinical utility supports the potential for PSMA as an imaging reporter.

Here we report the development of PSMA as a reporter-probe system for molecular-genetic imaging. PSMA was directly compared with 2 established reporter-probe systems using both in vitro and in vivo models. In a study using dynamic PET, the PSMA system had the highest signal-to-noise ratio of the 3 systems. The PSMA reporter was then applied to a mouse model to monitor hepatic adenoviral infection after intravenous injection by both optical and nuclear imaging. These results support the potential clinical utility of a PSMA-based reporter-probe system.

\section{MATERIALS AND METHODS}

Chemicals and primers were purchased from Sigma. Cell culture reagents were purchased from Mediatech, Inc., Gemini Bio-Products, and Invitrogen. Restriction enzymes and DNA-modifying enzymes were purchased from New England Biolabs. Plasmid and gel purification components were purchased from Qiagen, Inc., or Sigma. Transfection reagent was purchased from Invitrogen. Adenovirus 
purification kits were purchased from Puresyn, Inc. ${ }^{3} \mathrm{H}$-ganciclovir was purchased from Moravek Biochemicals. ${ }^{125} \mathrm{I}-\mathrm{NaI}$ was purchased from MP Biomedicals. The J591 antibody was kindly provided by Dr. Neil Bander (Weill Cornell Medical College, New, York, NY), and the HSV-sr39TK construct was provided by Dr. S. Sam Gambhir (Stanford University, Palo Alto, CA). Cell culture conditions, cloning of adenoviral vectors, and their validation are detailed in the supplemental data (available at http://jnm.snmjournals.org).

\section{Cell Uptake Assays}

Cellular uptake of ${ }^{125} \mathrm{I}$ was measured in a fashion similar to that applied by De la Vieja et al. (16), and HSV-sr39tk expression was measured as described by Yaghoubi and Gambhir (17). PSMA expression was assessed by measuring cellular uptake of the known, high-affinity PSMA-targeting ligand, 2-[3-[1-carboxy-5-(4-125I-iodo-benzoylamino)pentyl]-ureido]-pentanedioic acid (YC-I-27) (18). For uptake of ${ }^{125}$ I, cells were harvested in phosphate-buffered saline (PBS) using a cell scraper and placed in a fluorescence-activated cell sorter tube for quantification in an automated $\gamma$ counter (1282 Compugamma CS; Pharmacia/LKB Nuclear, Inc.). For ${ }^{3} \mathrm{H}$-ganciclovir, the amount of radiolabeled substrate retained was quantified by scintillation counting on a Microbeta Wallac (Perkin-Elmer). YC-I-27 uptake was also measured using the automated $\gamma$ counter. In each case, uptake was calculated as amount of radioactivity in the cells divided by radioactivity in the medium and normalized by the degree of expression of green fluorescent protein (GFP).

\section{In Vivo Imaging}

Studies were performed according to protocols approved by the Animal Care and Use Committee at Johns Hopkins University. A Matrigel (BD Biosciences) suspension model was developed to enable imaging of similar numbers of cells in vivo. Four- to 6-wk-old male athymic nude mice (Harlan Laboratories Inc.) were used for all experiments. Infected cells $\left(4 \times 10^{6}\right)$ were injected subcutaneously in the presence of Matrigel at a 2:1 (cell:Matrigel) ratio.

PET was performed for in vivo reporter comparison. 9-(4- ${ }^{18} \mathrm{~F}$-fluoro3 -[hydroxymethyl]butyl)guanine ( $\left.{ }^{18} \mathrm{~F}-\mathrm{FHBG}\right)$ and 2-(3-\{1-carboxy5-[(6- ${ }^{18} \mathrm{~F}$-fluoro-pyridine-3-carbonyl)-amino]-pentyl $\}$-ureido)-pentanedioic acid $\left({ }^{18} \mathrm{~F}-\mathrm{DCFPyL}\right)$ were synthesized as previously described $(19,20)$. ${ }^{124} \mathrm{I}-\mathrm{NaI}$ was kindly provided by Dr. Jason Lewis (Memorial SloanKettering Cancer Center, New York, NY). Athymic nude mice contralaterally implanted with reporter- or control-expressing Matrigel suspensions in the upper flanks were used for imaging. Mice were anesthetized with $3 \%$ isoflurane in oxygen for induction and were maintained under $1.5 \%$ isoflurane in oxygen at a flow rate of $0.8 \mathrm{~L} / \mathrm{min}$. Mice were then placed in the prone position on the gantry of an eXplore VISTA small-animal PET scanner (GE Healthcare Life Sciences) and injected intravenously with approximately $7.4 \mathrm{MBq}(200 \mu \mathrm{Ci})$ of each respective radiotracer in $200 \mu \mathrm{L}$ of PBS. Images were acquired as a dynamic scan of eighteen 5-min frames at $250-700 \mathrm{keV}$ for ${ }^{18} \mathrm{~F}$ and $400-700 \mathrm{keV}$ for ${ }^{124} \mathrm{I}$. Images were reconstructed using Fourier rebinning/2-dimensional ordered-subsets expectation maximization (1 iteration, 16 subsets), and the reconstruction included correction for radioactive decay, scanner dead time, and scattered radiation. After PET imaging, the mobile mouse holder was placed on the gantry of an X-SPECT (Gamma Medica-Ideas) small-animal imaging device to acquire the corresponding CT scan. PET and CT data were coregistered using AMIDE software (http://amide.sourceforge.net/).

To apply PSMA as a reporter by imaging transduced hepatocytes, near-infrared optical imaging and SPECT imaging were performed. Nude mice were injected intravenously with $1 \times 10^{7}$ infectious units of reporter adenovirus Ad-Track-PSMA or Ad-Track-luciferase, which were cloned using the AdEasy system (Agilent Technologies) in a $200-\mu \mathrm{L}$ volume. Luciferase was not used for imaging in this instance but as a control gene. For near-infrared imaging, $24 \mathrm{~h}$ after receiving
Ad-Track-PSMA or Ad-Track-luciferase, animals were administered 15 or $30 \mu \mathrm{g}$ of anti-PSMA antibody, J591 conjugated with IR800 in $200 \mu \mathrm{L}$ of PBS, via the lateral tail vein. For SPECT, animals received approximately 7.4 MBq $(200 \mu \mathrm{Ci})$ of the known, high-affinity PSMAtargeting ligand, 2-(3-[1-carboxy-5-[(5-125I-iodo-pyridine-3-carbonyl)amino]-pentyl]-ureido)-pentanedioic acid (YC-VI-11) (18) in $200 \mu \mathrm{L}$ of PBS also at $24 \mathrm{~h}$ after Ad-Track-PSMA or Ad-Track-luciferase. Animals received inhalational anesthesia (isoflurane) through a nose cone attached to the imaging bed. Near-infrared images were acquired $48 \mathrm{~h}$ after injection of conjugated J591 and imaged using the Pearl Impulse (LI-COR), whereas SPECT images were acquired after 15 min of radiopharmaceutical uptake using the X-SPECT. For SPECT, mice were scanned over $180^{\circ}$ in $5.5^{\circ}, 30$-s increments. SPECT images were coregistered with CT images. For each modality, images for AdTrack-PSMA or Ad-Track-luciferase were acquired at the same parameter settings and scaled to the same maximum values.

\section{Statistical Analysis}

Statistical analysis was performed using Prism software (GraphPad Software, Inc.). An unpaired, 2-tailed $t$ test was used, and $P$ values of less than 0.05 were considered significant.

\section{RESULTS}

\section{Radiochemical Syntheses}

Radiotracers used for PET, that is, ${ }^{18} \mathrm{~F}-\mathrm{FHBG},{ }^{124} \mathrm{I}-\mathrm{NaI},{ }^{18} \mathrm{~F}-$ DCFPyL, and $N$-[N-[(S)-1,3-dicarboxypropyl $]$ carbamoyl $]-4-{ }^{18} \mathrm{~F}-$ fluorobenzyl-L-cysteine ( $\left.{ }^{18} \mathrm{~F}-\mathrm{DCFBC}\right)(13)$, were synthesized at specific activities of $12.6 \pm 0.5 \mathrm{GBq} / \mu \mathrm{mol}(340 \pm 14 \mathrm{Ci} / \mathrm{mmol})$ with no carrier added, $43.7 \pm 24.6 \mathrm{GBq} / \mu \mathrm{mol}(1,180 \pm 665 \mathrm{Ci} /$ $\mathrm{mmol})$, and $786 \pm 542 \mathrm{GBq} / \mu \mathrm{mol}(21,233 \pm 14,651 \mathrm{Ci} / \mathrm{mmol})$, respectively. Despite the significant difference in specific activity between ${ }^{18} \mathrm{~F}-\mathrm{DCFPyL}$ and ${ }^{18} \mathrm{~F}-\mathrm{DCFBC}$, similar results were obtained on imaging.

\section{Adenoviral Reporter Comparison Model}

Our goal was to develop PSMA as an imaging reporter and to compare it equitably with established imaging reporters. Adenoviral vectors were applied for transgene expression for several reasons: as a means to transfer equal amounts of transgene reporters, because the adenovirus genome is maintained episomally and is not influenced by integration site or copy number, and because adenoviruses have been applied successfully in imaging reporter gene comparisons (21-23). Three putative reporter genes, PSMA, HSV-sr39tk, and hNIS, were subcloned downstream of a cytomegalovirus promoter in the E1 region of nonreplicating serotype 5 adenoviruses (Table 1). Each viral construct also expressed GFP for fluorescence detection of viral infection and titration. Two cell line models were applied to viral reporter studies, HCT116 colon cancer cells and PC3 prostate cancer cells engineered to express stably the coxsackie adenovirus receptor (PC3-CAR). The expression of each reporter was validated by Western blot analysis of infected HCT116 cells (multiplicity of infection, 1) $48 \mathrm{~h}$ after infection. Each reporter was detected at the appropriate molecular weight in infected cells, and no endogenous expression was visible under mock-infected or Ad-Track (GFP)infected control conditions (Fig. 1A). In addition, specific transgene delivery and expression were detected by a cell-based fluorescent immunoassay (Fig. 1A). Specifically, PC3-CAR cells were equally infected with each reporter adenovirus (multiplicity of infection, 500) and fixed $48 \mathrm{~h}$ after infection. Reporter protein expression was detected by primary antibody incubation followed by fluorescent secondary antibody staining and detection on a fluorescent 
TABLE 1

Description of Reporter Adenoviruses and Respective Imaging Modalities Used for Detection of Reporter Genes

\begin{tabular}{llc}
\hline \multicolumn{1}{c}{ Virus } & Reporter gene & Imaging modality \\
\hline Ad-Track-PSMA & Prostate-specific membrane antigen & Optical, PET, SPECT \\
Ad-Track-hNIS & Human sodium iodide symporter & PET, SPECT \\
Ad-HSV-sr39tk & Herpes simplex virus mutant thymidine kinase & PET \\
\hline
\end{tabular}

plate reader. The results confirm homogeneous transgene expression and specificity by immunodetection.

\section{In Vitro Functionality of Reporter Vectors}

Functional assays for each reporter were analyzed using HCT116 and PC3-CAR cell lines. Cells were infected with each reporter adenovirus. After $48 \mathrm{~h}$, the level of adenovirus infection was quantified by GFP fluorescence and the level of imaging reporter activity was quantified by functional binding or uptake of radiolabeled probes. Specific assay conditions were applied for each reporter gene because of the different mechanisms of reporter probe binding or uptake. Conditions were based on previously

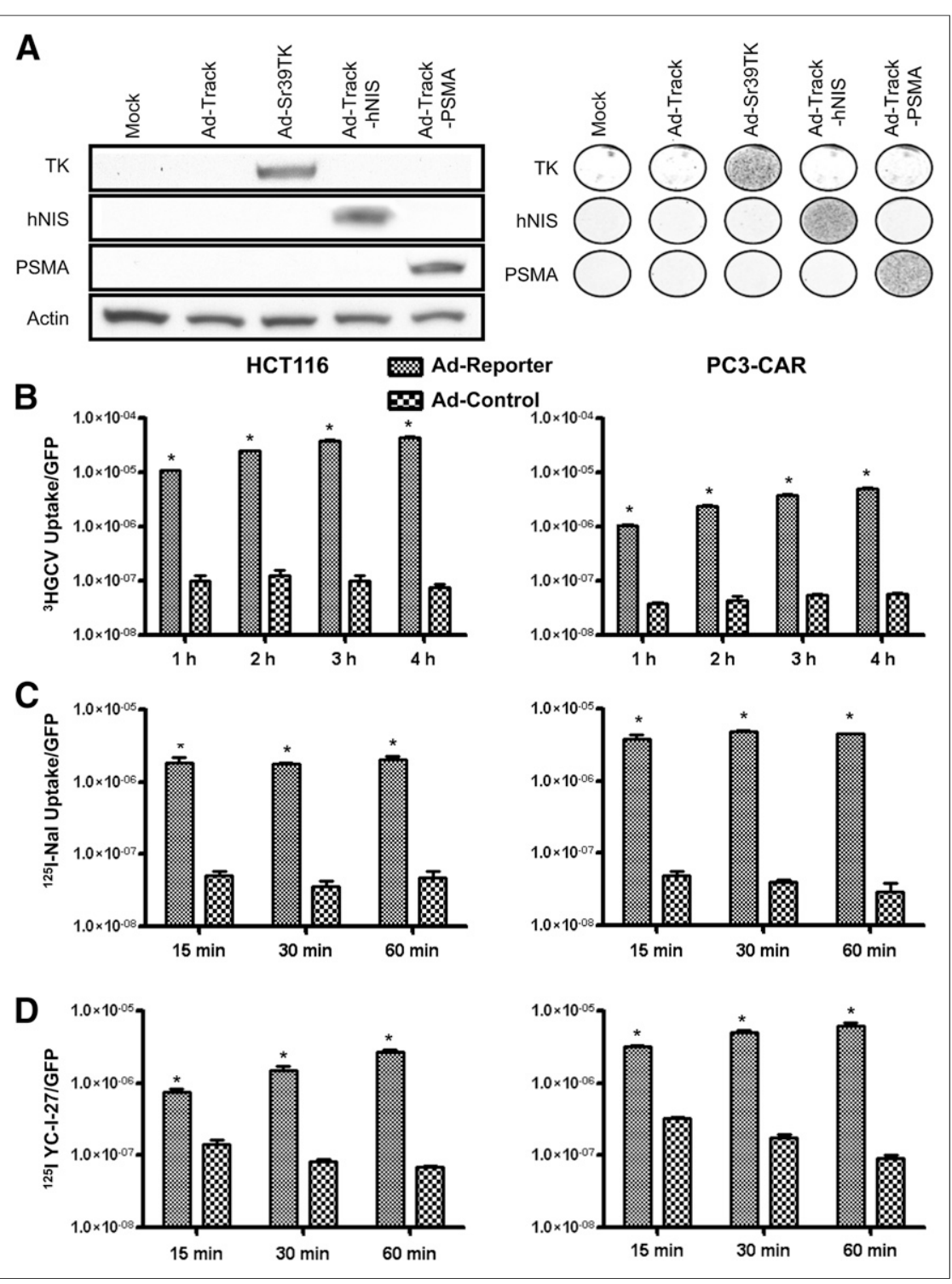

FIGURE 1. In vitro characterization of reporter adenovirus. (A) Reporter expression was validated by Western blot in HCT116 cells and by fluorescent cellular immunoassay in PC3-CAR cells $48 \mathrm{~h}$ after infection of reporter adenovirus. Supplemental data provide experimental details. (B-D) Functionality of each reporter was verified in HCT116 and PC3-CAR cells by ${ }^{3} \mathrm{H}$-ganciclovir (HGCV) uptake (B), ${ }^{125}$ I uptake (C), and YC-I-27 binding (D). Data were normalized to cellular GFP expression and plotted as mean $\pm \mathrm{SE}$ of mean of 3 replicates. ${ }^{*} P<0.01$. established assay conditions both in our own laboratory and in the literature $(16,17)$. For ${ }^{3} \mathrm{H}$-ganciclovir uptake, a prolonged washout period was not performed and as a result this assay does not differentiate between phosphorylated ${ }^{3} \mathrm{H}$-ganciclovir and unphosphorylated ${ }^{3} \mathrm{H}$-ganciclovir that is associated with the cells at the time of harvest. YC-I-27 uptake in PSMA-infected cells proved more time-dependent than the corresponding radiotracers were for NIS or HSV-sr39tk. We hypothesize that this may be due to the mechanism of sequestration, whereby ${ }^{124} \mathrm{I}-\mathrm{NaI}$ and ${ }^{3} \mathrm{H}$-ganciclovir are able to diffuse passively or are transported into cells where they are trapped, whereas YC-I-27 can enter the cell only via binding and internalization of PSMA at a 1:1 stoichiometry. Despite those caveats, in all cases specific and significant uptake was observed in cells infected with reporter adenovirus, resulting in a signal-to-noise ratio of at least 10 - to 20 -fold relative to control adenovirus (Figs. 1B-1D).

\section{In Vivo Comparison of Imaging Reporters}

To compare reporter-probe systems in vivo, we developed a Matrigel suspension model (Supplemental Fig. 1). In this model, PC3-CAR cells were infected in vitro with an equal multiplicity of infection of a PET imaging reporter or control adenovirus (Ad-Track-luciferase) and then each population of infected cells was separately mixed with Matrigel and implanted contralaterally into the upper flanks of a nude mouse ( $24 \mathrm{~h}$ after infection). Twenty-four hours after implantation, in vivo GFP imaging was performed to ensure that a similar level of control and reporter-infected cells remained at the sites of implantation. The contralateral cell masses were generally within $30 \%$ as measured by GFP imaging in vivo (Supplemental Fig. 2). The next day, the animals were subjected to a dynamic PET 
study whereby approximately $7.4 \mathrm{MBq}(200 \mu \mathrm{Ci})$ of each respective radiotracer was administered intravenously and cellular uptake was quantified for $90 \mathrm{~min} .{ }^{18} \mathrm{~F}-\mathrm{FHBG}$ was applied for HSV-sr39tk imaging, ${ }^{124} \mathrm{I}-\mathrm{NaI}$ for hNIS imaging, and ${ }^{18} \mathrm{~F}-\mathrm{DCFPyL}$ for PSMA imaging $(3,20,24)$. This model was used for comparing the reporter-probe systems for 3 reasons: first, in vitro infection allowed equal transgene delivery of reporters and direct evaluation of implantation by GFP imaging; second, the application of a relatively small number of cells and the short duration of the experiment minimized the influence of biologic events, such as cell growth or substantial development of tumor vasculature, on imaging reporter activity; and third, the reporter virus was compared with a control virus within the same

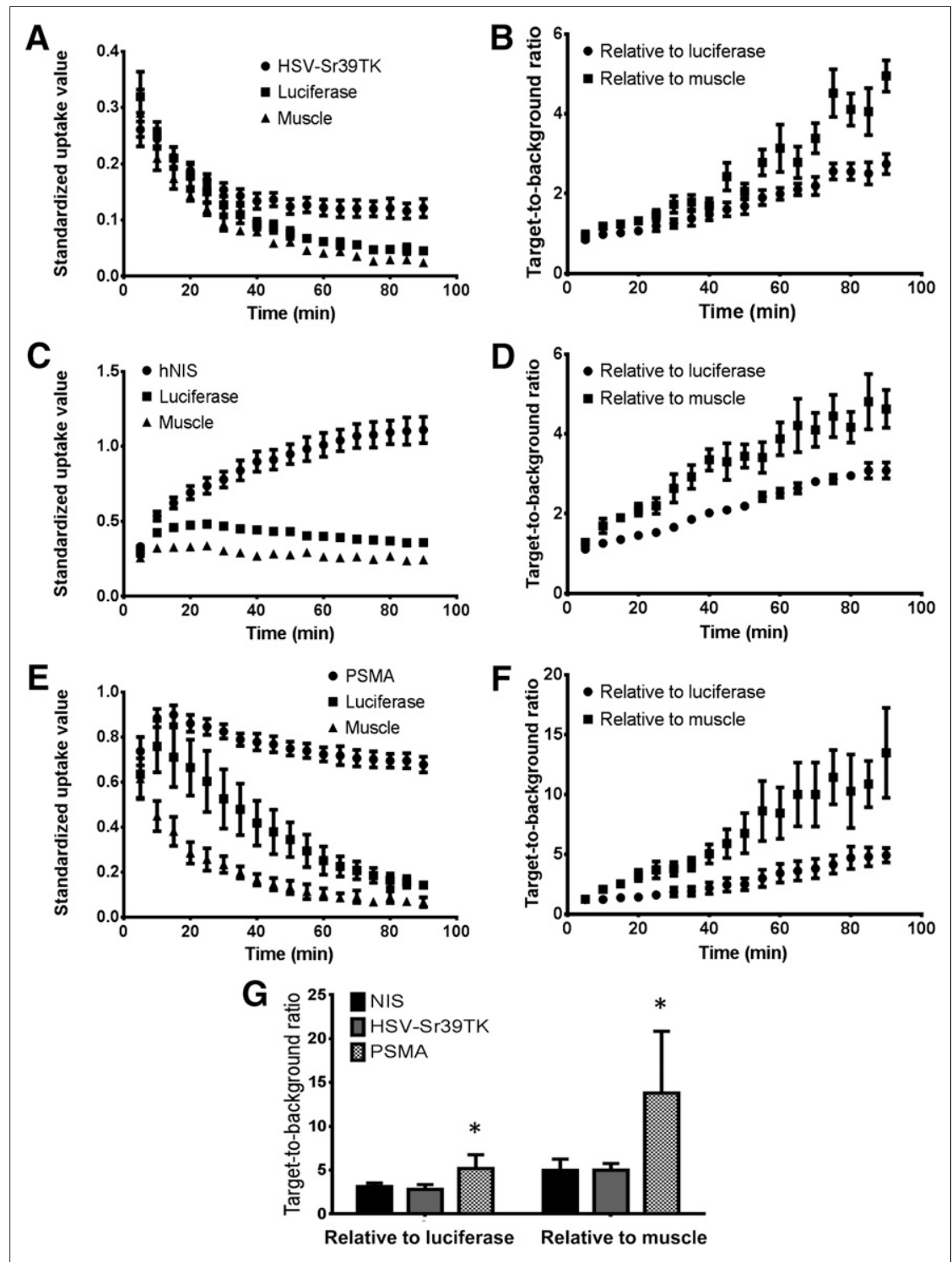

FIGURE 2. In vivo comparison of imaging reporters. Mice were contralaterally implanted with 2 Matrigel suspensions of cells expressing either reporter or negative control gene (luciferase) in upper flanks. Luciferase was not used for imaging but as a control gene. After $48 \mathrm{~h}$, each radiotracer was injected and uptake was quantified from regions of interest drawn around the reporter, luciferase control, and muscle. Graphs illustrate time-activity curves for respective reporter-probe systems in the Matrigel suspension model for HSV-sr39tk: ${ }^{18} \mathrm{~F}-\mathrm{FHBG}(\mathrm{A}), \mathrm{hNIS}:{ }^{125}$ (C), and PSMA: ${ }^{18} \mathrm{~F}-\mathrm{DCFPyL}(\mathrm{E})$ and reporter-derived signal-to-control and signal-to-muscle ratios for HSV-sr39tk (B), hNIS (D), and PSMA (F) systems, as well as comparison of peak signal-to-noise ratios of each reporter-probe pair (G). Data are mean \pm SE of mean of 4 animals. ${ }^{*} P<0.05$. animal, providing an additional control to obviate animal-to-animal variation.

Kinetic uptake of each imaging reporter in target and control tissue was represented as a standardized uptake value or standardized uptake value relative to control tissue (Fig. 2). For the HSV-sr39tk reporter, high initial uptake was observed in all tissues followed by rapid clearance from cells. HSV-sr39tk-specific setion of the radiotracer was observed after 30-40 min. The the compound into the cells, with the unphosphorylated radiotracer diffusing out of the cells but phosphorylated radiotracer being retained. A peak target-to-background ratio of $2.81 \pm$ 0.27 relative to control cells and $4.99 \pm$ 0.38 relative to muscle was observed (Fig. $2 \mathrm{G})$. The signal accumulation in hNISexpressing cells occurred quickly and plateaued roughly 80-90 min after injection (Fig. 2C). No significant uptake was observed in control tissue. A peak target-tobackground ratio of $3.13 \pm 0.20$ relative to control cells and $4.96 \pm 0.65$ relative to muscle was observed (Fig. 2G). These results are similar to those described by Miyagawa, who compared hNIS and HSV-sr39tk reporter gene systems in a cardiac imaging model (22). Despite differences in the mechanism of reporter probe accumulation, both the maximum signal-to-background ratios and the maximum percentage injected dose per cubic centimeter of tissue of these $2 \mathrm{im}$ aging reporters were similar. This finding supports the utility of the Matrigel suspension model as a means to compare PSMA with the existing reporter-probe systems.

The kinetics of the PSMA imaging reporter using ${ }^{18} \mathrm{~F}-\mathrm{DCFPyL}$ displayed rapid probe uptake, with the signal peaking 15 min after injection, followed by a plateau shortly thereafter (Fig. 2E). Clearance from control tissue occurred more slowly for ${ }^{18} \mathrm{~F}-\mathrm{DCFPyL}$ than for the radioligands used for the hNIS and HSV-sr39tk systems. A peak target-to-background ratio of $5.19 \pm$ 0.78 relative to control cells and $13.80 \pm$ 3.52 relative to muscle was observed (Fig. $2 \mathrm{G})$. The maximum-intensity projection images, cropped above the liver and below the thyroid for each reporter-probe system, demonstrate the specific uptake in the reporter-expressing cells when compared with the muscle or with control Matrigel suspension (Fig. 3A). The target-to-background profiles revealed that a signal-tonoise ratio of greater than 2 (relative to muscle) could be detected after $45 \mathrm{~min}$ for HSV-sr39tk, $20 \mathrm{~min}$ for hNIS, and 10 min for PSMA (Figs. 2B, 2D, and 2F).

Maximum signal-to-noise ratios were observed at $90 \mathrm{~min}$, with the PSMA system having the highest ratio relative both to the luciferase Matrigel suspension and to the 

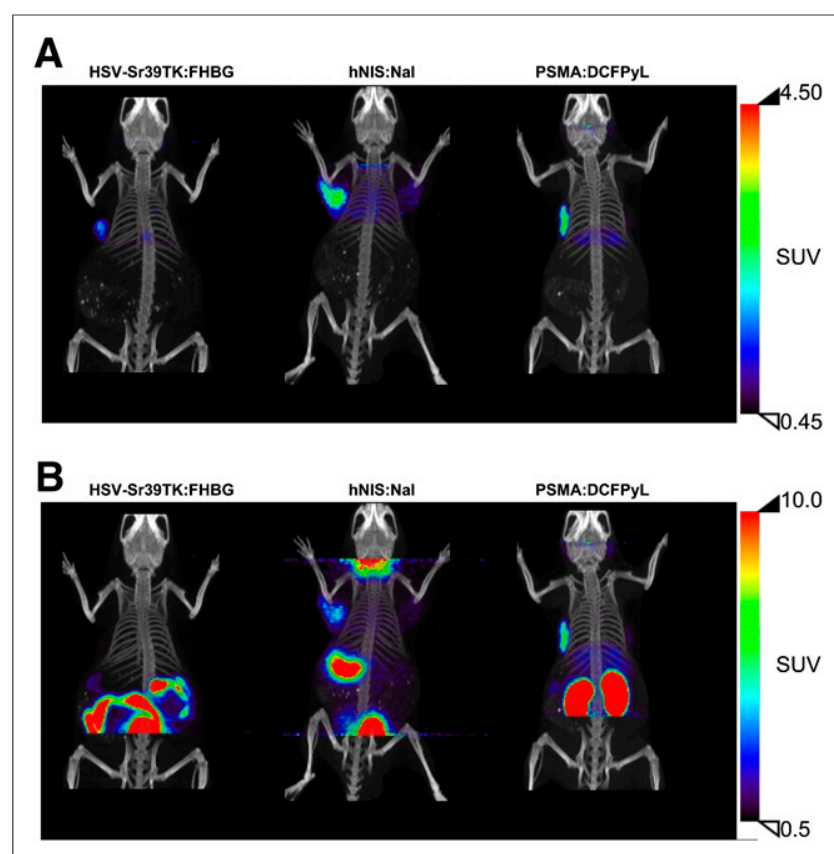

FIGURE 3. PET/CT imaging of reporter-probe systems. Representative maximum-intensity projections of reporter-probe systems of data collected from 60 to $90 \mathrm{~min}$ after radiotracer injection. (A) Images cropped above liver and below thyroid display the ability of each reporter to sequester its respective probe. (B) Uncropped images demonstrate background associated with each reporter-probe system. All images were decay-corrected and scaled to the same maximum value.

muscle (Fig. 2G). Uncropped images from each scan highlight differences in the efficiency of the reporter to sequester its cognate probe and demonstrate background due to natural expression of reporter genes or probe metabolism (Fig. 3B). High gastrointestinal uptake was observed in the HSV-sr39tk system, which has previously been attributed to probe metabolism (25). Background signal from the stomach and thyroid was observed in the hNIS system because of natural expression of hNIS in these tissues. For PSMA, kidney uptake was observed in part because of renal clearance, as well as because of PSMA expression in the proximal tubules (26). The PSMA reporter-probe system was also evaluated using ${ }^{18} \mathrm{~F}$-DCFBC, which has been administered to patients $(13,27)$. Results from ${ }^{18} \mathrm{~F}$-DCFBC were similar to results obtained with ${ }^{18} \mathrm{~F}-\mathrm{DCFPyL}$, with the exception of a slower clearance from the control Matrigel suspension (Supplemental Fig. 3).

\section{Application of the PSMA Reporter-Probe System}

It has been previously reported in rodent models that adenoviral vectors administered via systemic injection will result in hepatocyte infection and transgene expression (28). Optical imaging of mouse livers was performed $72 \mathrm{~h}$ after infection and $48 \mathrm{~h}$ after administration of 15 or $30 \mu \mathrm{g}$ of J591-IR800 (Fig. 4A). Regions of interest were drawn around the liver, and a signal-to-noise ratio of approximately 3:1 was observed for the PSMA imaging reporter relative to control adenovirus. This model was also applied to PSMA-specific imaging with SPECT using YC-VI-11 (18), with similar results, with a signal-to-noise ratio of approximately $2: 1$ being observed 15 min after administration of radiotracer (Fig. 4B). Collectively, these results reveal PSMA to be a sensitive and specific imaging reporter that can be applied to multiple imaging modalities.

\section{DISCUSSION}

We report the capacity of PSMA to serve as an imaging reporter. For comparison, 2 established and clinically applied imaging systems were used. Although PSMA was found to have superior signal in these studies, the overall signal intensities were comparable. Accordingly, one may select any of these 3 imaging reporter systems after weighing the advantages and disadvantages of each. One must also consider the potential for each of these reporter systems to be improved. The HSV-tk-based reporter-probe system, for example, has undergone multiple iterations that have improved the sensitivity of detection; however, immunogenicity, probe metabolism, and pharmacokinetics (in humans) of the administered radioactive nucleoside analogs, particularly in the periphery, remain as obstacles. To overcome these obstacles partially, systems based on human thymidine kinase have been engineered and are currently undergoing testing $(5,7,29)$. A hurdle in using hNIS as a reporter for imaging is the background expression from tissues such as the thyroid and stomach, which naturally express hNIS, as well as a limited imaging window due to rapid iodide (for PET) efflux with exogenous expression of hNIS (30). PSMA imaging reporter studies would also have background signal in kidney and in patients with prostate or possibly other cancers (31), although agents under development clear significantly more rapidly from kidney than from tumor (32).

Enzymatic and transporter-based reporter genes are able to amplify the signal by accumulation of probe, whereas receptorbased reporters can yield only a 1:1 stoichiometry of probe to reporter gene molecule. The PSMA probes evaluated in our study, as well as other probes in clinical and preclinical development, bind to the active site of PSMA on the surface of the cell. After

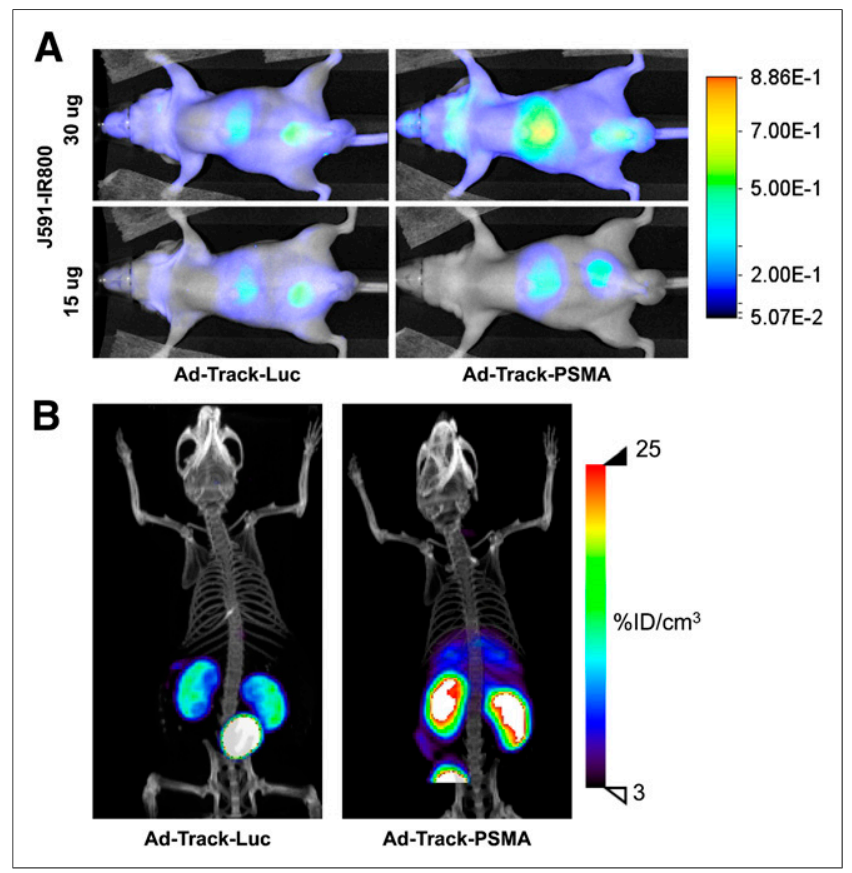

FIGURE 4. Monitoring hepatic infection and transgene expression by imaging PSMA in a murine model. Athymic nude mice were intravenously administered adenovirus expressing PSMA or negative control gene (luciferase [Luc]). Seventy-two hours after injection of adenovirus, animals were imaged and specific uptake was observed in liver of animals by optical (A) and SPECT (B) imaging. SPECT images were obtained $15 \mathrm{~min}$ after injection of radiotracer, with similar levels of radioactivity in liver at $4 \mathrm{~h}$ after injection (Supplemental Fig. 4). 
ligand binding, PSMA can internalize, suggesting a means for signal retention and amplification. To date, the enzymatic or transporter properties of PSMA have not been exploited for such signal amplification. Nevertheless, our data suggest that ligand binding alone is sufficient for detection and yields a higher signal-to-noise ratio than the hNIS and HSV-sr39tk reporterprobe systems.

Finally, one must consider the biologic effect of the reporter gene on the cell or tissue of interest. To date, there have been no significant negative effects of HSV-tk or hNIS transgene expression. In our hands, PSMA expression has not significantly altered cellular phenotype in vitro in any of multiple mammalian cell types. However, the functions of PSMA in prostate cancer and the neovasculature of many solid tumors are unclear. In the brain, PSMA cleaves $N$-acetylaspartylglutamate to liberate glutamate as a neurotransmitter, and in the intestine PSMA is involved in folate metabolism (33). A study on retinal angiogenesis suggested PSMA plays a vascular endothelial growth factor-independent role in ocular neovascularization (34). Data from Yao et al. also suggest that PSMA expression increases folate uptake and proliferation in prostate cancer cells (35). Accordingly, further experimentation is warranted to determine the effect of exogenous PSMA expression on cells. Functional modifications that alter PSMA enzymatic activity, ligand-binding ability, and cellular internalization have been identified and could be used if negative effects of transgene expression are discovered $(36,37)$.

\section{CONCLUSION}

We have tested PSMA, a prostate cancer biomarker located on the cell membrane that possesses receptor, transporter, and enzymatic functions, as an imaging reporter. PSMA was compared with 2 reporter-probe systems, hNIS and HSV-sr39tk, in an in vivo model. The results demonstrate that PSMA is able to sequester imaging ligands with high affinity and with a high target-tobackground ratio. Additional studies support PSMA as an imaging reporter with PET, SPECT, and optical imaging modalities. As advances in cell engineering and gene therapies emerge, PSMA may provide a sensitive and nonimmunogenic reporter by which gene and cell therapies can be monitored in vivo, noninvasively.

\section{DISCLOSURE}

The costs of publication of this article were defrayed in part by the payment of page charges. Therefore, and solely to indicate this fact, this article is hereby marked "advertisement" in accordance with 18 USC section 1734. This work was supported by CA134675, EB5324, and GM79838. No other potential conflict of interest relevant to this article was reported.

\section{ACKNOWLEDGMENTS}

We thank Gilbert Green for providing assistance with small-animal imaging, and we thank Mrudula Pullambhatla, Sridhar Nimmagadda, and Catherine Foss for providing reagents and assistance with in vitro functional assays.

\section{REFERENCES}

1. Brader P, Serganova I, Blasberg RG. Noninvasive molecular imaging using reporter genes. J Nucl Med. 2013;54:167-172.
2. Barton KN, Stricker H, Brown SL, et al. Phase I study of noninvasive imaging of adenovirus-mediated gene expression in the human prostate. Mol Ther. 2008; 16:1761-1769.

3. Yaghoubi S, Barrio JR, Dahlbom M, et al. Human pharmacokinetic and dosimetry studies of $\left[{ }^{18} \mathrm{~F}\right] \mathrm{FHBG}$ : a reporter probe for imaging herpes simplex virus type-1 thymidine kinase reporter gene expression. J Nucl Med. 2001;42:12251234.

4. Beeres SL, Bengel FM, Bartunek J, et al. Role of imaging in cardiac stem cell therapy. J Am Coll Cardiol. 2007;49:1137-1148.

5. Campbell DO, Yaghoubi SS, Su Y, et al. Structure-guided engineering of human thymidine kinase 2 as a positron emission tomography reporter gene for enhanced phosphorylation of non-natural thymidine analog reporter probe. J Biol Chem. 2012;287:446-454.

6. Likar Y, Zurita J, Dobrenkov K, et al. A new pyrimidine-specific reporter gene: a mutated human deoxycytidine kinase suitable for PET during treatment with acycloguanosine-based cytotoxic drugs. J Nucl Med. 2010;51: 1395-1403.

7. Yaghoubi SS, Campbell DO, Radu CG, Czernin J. Positron emission tomography reporter genes and reporter probes: gene and cell therapy applications. Theranostics. 2012;2:374-391.

8. Liu H, Rajasekaran AK, Moy P, et al. Constitutive and antibody-induced internalization of prostate-specific membrane antigen. Cancer Res. 1998;58:40554060.

9. Rajasekaran AK, Anilkumar G, Christiansen JJ. Is prostate-specific membrane antigen a multifunctional protein? Am J Physiol Cell Physiol. 2005;288:C975C981.

10. Mlcochová P, Barinka C, Tykvart J, Sácha P, Konvalinka J. Prostate-specific membrane antigen and its truncated form PSM'. Prostate. 2009;69:471-479.

11. Afshar-Oromieh A, Malcher A, Eder M, et al. PET imaging with a $\left[{ }^{68} \mathrm{Ga}\right] g a l-$ lium-labelled PSMA ligand for the diagnosis of prostate cancer: biodistribution in humans and first evaluation of tumour lesions. Eur J Nucl Med Mol Imaging. 2013;40:486-495.

12. Barrett JA, Coleman RE, Goldsmith SJ, et al. First-in-man evaluation of 2 highaffinity PSMA-avid small molecules for imaging prostate cancer. $\mathrm{J} \mathrm{Nucl} \mathrm{Med}$. 2013;54:380-387.

13. Cho SY, Gage KL, Mease RC, et al. Biodistribution, tumor detection, and radiation dosimetry of ${ }^{18} \mathrm{~F}$-DCFBC, a low-molecular-weight inhibitor of prostatespecific membrane antigen, in patients with metastatic prostate cancer. $J$ Nucl Med. 2012;53:1883-1891.

14. Chen Y, Dhara S, Banerjee SR, et al. A low molecular weight PSMA-based fluorescent imaging agent for cancer. Biochem Biophys Res Commun. 2009;390: 624-629.

15. Banerjee SR, Pullambhatla M, Byun Y, et al. Sequential SPECT and optical imaging of experimental models of prostate cancer with a dual modality inhibitor of the prostate-specific membrane antigen. Angew Chem Int Ed Engl. 2011;50: 9167-9170.

16. De la Vieja A, Ginter CS, Carrasco N. Molecular analysis of a congenital iodide transport defect: G543E impairs maturation and trafficking of the Na+/I- symporter. Mol Endocrinol. 2005;19:2847-2858.

17. Yaghoubi SS, Gambhir SS. Measuring herpes simplex virus thymidine kinase reporter gene expression in vitro. Nat Protoc. 2006;1:2137-2142.

18. Chen Y, Foss CA, Byun Y, et al. Radiohalogenated prostate-specific membrane antigen (PSMA)-based ureas as imaging agents for prostate cancer. J Med Chem. 2008;51:7933-7943.

19. Ponde DE, Dence CS, Schuster DP, Welch MJ. Rapid and reproducible radiosynthesis of $\left[{ }^{18} \mathrm{~F}\right]$ FHBG. Nucl Med Biol. 2004;31:133-138.

20. Chen Y, Pullambhatla M, Foss CA, et al. 2-(3- $\left\{1\right.$-carboxy-5-[(6- $\left[{ }^{18}\right.$ F $]$ fluoro-pyridine-3-carbonyl)-amino]-pentyl $\}$-ureido)-pentanedioic acid, $\left[{ }^{18} \mathrm{~F}\right] \mathrm{DCFPyL}$, a PSMA-based PET imaging agent for prostate cancer. Clin Cancer Res. 2011; 17:7645-7653.

21. Gil JS, Machado HB, Herschman HR. A method to rapidly and accurately compare the relative efficacies of non-invasive imaging reporter genes in a mouse model and its application to luciferase reporters. Mol Imaging Biol. 2012;14: $462-471$.

22. Min JJ, Iyer M, Gambhir SS. Comparison of $\left[{ }^{18} \mathrm{~F}\right] \mathrm{FHBG}$ and $\left[{ }^{14} \mathrm{C}\right] \mathrm{FIAU}$ for imaging of HSV1-tk reporter gene expression: adenoviral infection vs stable transfection. Eur J Nucl Med Mol Imaging. 2003;30:1547-1560.

23. Miyagawa M, Anton M, Wagner B, et al. Non-invasive imaging of cardiac transgene expression with PET: comparison of the human sodium/iodide symporter gene and HSV1-tk as the reporter gene. Eur J Nucl Med Mol Imaging. 2005;32:1108-1114.

24. Dingli D, Kemp BJ, O’Connor MK, Morris JC, Russell SJ, Lowe VJ. Combined I-124 positron emission tomography/computed tomography imaging of NIS gene expression in animal models of stably transfected and intravenously transfected tumor. Mol Imaging Biol. 2006;8:16-23. 
25. Tjuvajev JG, Doubrovin M, Akhurst T, et al. Comparison of radiolabeled nucleoside probes (FIAU, FHBG, and FHPG) for PET imaging of HSV1-tk gene expression. J Nucl Med. 2002;43:1072-1083.

26. Bacich DJ, Pinto JT, Tong WP, Heston WD. Cloning, expression, genomic localization, and enzymatic activities of the mouse homolog of prostate-specific membrane antigen/NAALADase/folate hydrolase. Mamm Genome. 2001;12:117-123.

27. Mease RC, Dusich CL, Foss CA, et al. N-[N-[(S)-1,3-dicarboxypropyl]carbamoyl]-4-[ $\left.{ }^{18} \mathrm{~F}\right]$ fluorobenzyl-L-cysteine, $\left[{ }^{18} \mathrm{~F}\right] \mathrm{DCFBC}$ : a new imaging probe for prostate cancer. Clin Cancer Res. 2008;14:3036-3043.

28. Johnson M, Huyn S, Burton J, Sato M, Wu L. Differential biodistribution of adenoviral vector in vivo as monitored by bioluminescence imaging and quantitative polymerase chain reaction. Hum Gene Ther. 2006;17:1262-1269.

29. Ponomarev V, Doubrovin M, Shavrin A, et al. A human-derived reporter gene for noninvasive imaging in humans: mitochondrial thymidine kinase type $2 . \mathrm{J} \mathrm{Nucl}$ Med. 2007;48:819-826.

30. Haberkorn U. Gene therapy with sodium/iodide symporter in hepatocarcinoma. Exp Clin Endocrinol Diabetes. 2001;109:60-62.

31. Chang SS, O'Keefe DS, Bacich DJ, Reuter VE, Heston WD, Gaudin PB. Prostate-specific membrane antigen is produced in tumor-associated neovasculature. Clin Cancer Res. 1999;5:2674-2681.
32. Banerjee SR, Pullambhatla M, Shallal H, Lisok A, Mease RC, Pomper MG. A modular strategy to prepare multivalent inhibitors of prostate-specific membrane antigen (PSMA). Oncotarget. 2011;2:1244-1253.

33. Heston WD. Characterization and glutamyl preferring carboxypeptidase function of prostate specific membrane antigen: a novel folate hydrolase. Urology. 1997;49(3A suppl):104-112.

34. Grant CL, Caromile LA, Ho V, et al. Prostate specific membrane antigen (PSMA) regulates angiogenesis independently of VEGF during ocular neovascularization. PLOS ONE. 2012;7:e41285.

35. Yao V, Berkman CE, Choi JK, O'Keefe DS, Bacich DJ. Expression of prostatespecific membrane antigen (PSMA), increases cell folate uptake and proliferation and suggests a novel role for PSMA in the uptake of the non-polyglutamated folate, folic acid. Prostate. 2010;70:305-316.

36. Anilkumar G, Rajasekaran SA, Wang S, Hankinson O, Bander NH, Rajasekaran AK. Prostate-specific membrane antigen association with filamin A modulates its internalization and NAALADase activity. Cancer Res. 2003;63: 2645-2648.

37. Rajasekaran SA, Anilkumar G, Oshima E, et al. A novel cytoplasmic tail MXXXL motif mediates the internalization of prostate-specific membrane antigen. Mol Biol Cell. 2003; $14: 4835-4845$. 